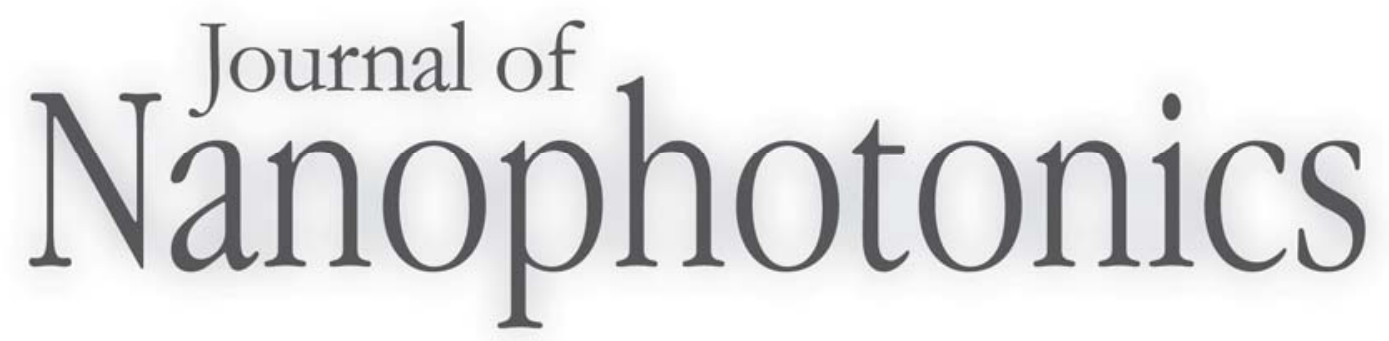

SPIEDigitalLibrary.org/jnp

\title{
Commentary: Raman nanospectroscopy of single DNA molecules
}

Dmitri Petrov

\section{O SPIE}




\section{Commentary: Raman nanospectroscopy of single DNA molecules}

\section{Dmitri Petrov}

ICFO - Institut de Ciencies Fotoniques, Mediterranean Technology Park, 08860, Castelldefels (Barcelona), Spain and ICREA - Institució Catalana de Recerca i Estudis Avançats, 08010,

Barcelona, Spain

Dmitri.Petrov@icfo.es

Among biological molecules a deoxyribonucleic acid (DNA) is the most important one because it is found in all living organisms. DNA is often referred to as a double helix because of its appearance. DNA is made of two long strands called nucleotides that run in opposite directions from one another. Nucleotides are made of sugars and phosphate groups that are joined together by ester bonds. Attached to each of the sugars is a molecule called a base. Four different types of bases encode the information that is used for cell replication. Among current DNA detection techniques the Raman scattering is the most powerful tool for gaining information on the chemical structure of the molecule. Raman study of DNA molecules or their basic constituents has a long history since 80th (for example, [1] ) and has provided a valuable information on this molecule, however the ultimate goal of Raman measurements has been to work with a minimal concentration of the molecules in a sample, ideally to obtain Raman signals from a single molecule. A single-molecule approach makes it possible to explore individual molecular details that cannot be obtained by ensemble-averaged results. The main obstacle to achieve this aim is the inherit low efficiency of the Raman scattering.

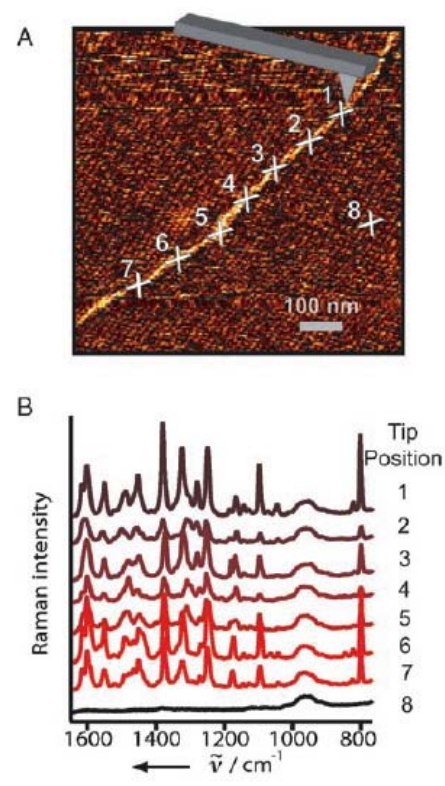

Fig. 1. A) Topography image showing seven adjacent spots corresponding to the positions of the TERS experiments and one additional spot for the reference measurement (position 8). B) The Raman spectra of the positions in (A). (From Ref. 6). 
Previously a strong improvement of the Raman spectroscopy was achieved conducting measurements of concentrated solutions of the DNA bases on rough metal (silver or gold) surfaces [2] or adding metal colloids to the samples [3]. The key element of these measurements is the Surface-Enhancement-Raman-Scattering (SERS) - a considerable enhancement of the emitted signal due to coupling of electromagnetic fields with optically excited local surface plasmons in nanosized metal structures as well as the so-called charge transfer enhancement generated by the formation of metal-ligand complexes [4]. Recently a Tip-Enhanced-RamanScattering (TERS), when a rough metal surface is concentrating on a small region directly at the apex of a scanning probe tip, also provided Raman measurements of DNA components [5].

Experiments showed that now SERS and TERS may permit one to obtain Raman signals of single DNA molecules. A single-molecule nature of these signals is usually proved by estimations of the number of molecules that might be in the confocal volume. Recently several studies showed directly that the Raman signal is originated from a single DNA molecule. To do this, in Ref. 6 the single DNA was stuck to a surface in order to realize the excitation in a TERS configuration (Fig. 1). The excitation beam was focussed onto a silver-coated tip, while the sample is moved independently. This experiment gives high sensitivity at lateral resolution down to a few tens of nucleobasis. However, this leaves the DNA in a static unnatural state which is sufficient for DNA sequence studies but still leaves a space for possible speculations on effects of the substrate induced mechanical tension.

To overcome the problem of immobilization of the DNA molecule without its attaching to a surface an additional optical technique - optical tweezers - was implemented in [7]. The molecule studied was double-stranded $\lambda$-DNA amplified at $12 \mathrm{kbp}$ from E. coli using standard polymerase chain reaction (PCR) techniques. The molecules were tagged with biotin and dioyxgenin at each end in order to attach to streptavidin and ant-dioxygenin (anti-DIG) coated polystyrene beads, respectively. Two focussed optical beams held the beads and aligned the DNA such that the Raman excitation beam passed between the beads (see Fig. 2).

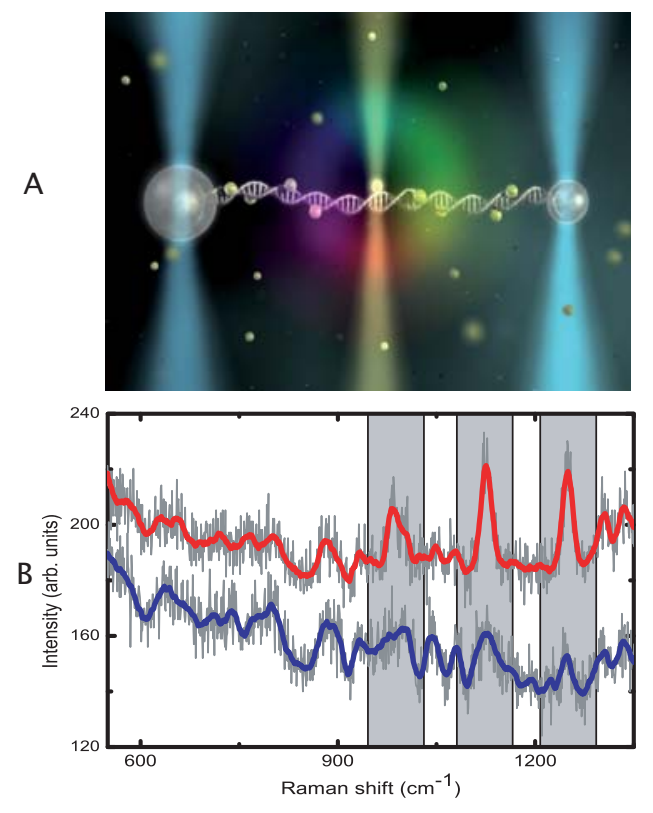

Fig. 2. A) The experimental setup. B) A spectrum (top plot) from a typical SERS measurement of a single DNA molecule plotted against a background spectrum (bottom plot). (From Ref. 7).

Silver colloids (the particle diameter about $70 \mathrm{~nm}$ ) was added. The colloids bind nonspecific 
to the negatively charged DNA molecules and permit to achieve the SERS. To verify that the Raman signals originate from a single DNA molecule, force-extension curves were measured of the DNA molecules that produced a Raman signal. To measure the background signal is also very easy in this experimental configuration by moving one of the trapped bead and hence, moving the DNA molecule out of the confocal volume. This technique of the single molecule DNA detection offers distinct advantages over the current methods of studying single DNA spectroscopically that require the DNA to be stuck to a surface. One can envision with the current configuration correlating DNA Raman signal with the force sensing and manipulation aspects of the optical trap. This could add a new dimension to dynamical studies of structural conformation, such as extension or twisting, or even protein diffusion along the DNA.

However, further studies must be performed that consider the DNA-metal interaction in order to shed more light on the specific conformations of DNA structure at the metal surface. A combination of optical trapping technique with TERS also looks very promising because Raman spectra spatial resolved along a single DNA molecule may be obtained.

\section{References}

[1] B. Prescott, W. Steinmetz, and G. J. Thomas Jr., "Characterization of DNA structures by laser Raman spectroscopy," Biopolymers 23, 235-256 (1984) [doi:10.1002/bip.360230206].

[2] C. Otto, T. J. J. van den Tweel, F. F. M. de Mul, and J. Greve, "SurfaceEnhanced Raman Scattering of DNA bases," J. Raman Spectroscopy 17, 289-298 (1986) [doi:10.1002/jrs.1250170311].

[3] K. Kneipp, H. Kneipp, V. B. Kartha, R. Manoharan, G. Deinum, I. Itzkan, R. R. Dasari, and M. S. Feld, "Detection and identification of a single DNA base molecule using surface-enhanced Raman scattering (SERS)," Phys. Rev. E 57, R6281-R6284 (1998) [doi:10.1103/PhysRevE.57.R6281].

[4] E. L. Ru and P. Etchegoin, Principles of Surface-Enhanced Raman Spectroscopy and Related Plasmonic Effects, Elsevier, Amsterdam (2009).

[5] A. Rasmussen and V. Deckert, "Surface- and tip-enhanced Raman scattering of DNA components," J. Raman Spectroscopy 37, 311-317 (2006) [doi:10.1002/jrs.1480].

[6] E. Bailo and V. Deckert, "Tip-enhanced Raman spectroscopy of single RNA strands: towards a novel direct-sequencing method," Angew. Chem. Int. Ed. 47, 1658-1661 (2008) [doi:10.1002/anie.200704054].

[7] S. Rao, S. Raj, S. Balint, C. B. Fons, S. Campoy, M. Llagostera, and D. Petrov, "Single DNA molecule detection in an optical trap using surface-enhanced Raman scattering," Appl. Phys. Lett. 96, 213701 (2010) [doi:10.1063/1.3431628]. 